\title{
Simulation of disc-bulge-halo galaxies using parallel GPU based codes
}

\author{
O. Veles ${ }^{1,3}$, P. Berczik ${ }^{2,1,3}$ and A. Just ${ }^{3}$ \\ ${ }^{1}$ Main Astronomical Observatory, National Academy of Sciences of Ukraine, \\ 27 Akademika Zabolotnoho St., 03680, Kyiv, Ukraine \\ email: veles@mao.kiev.ua \\ ${ }^{2}$ National Astronomical Observatories of China, Chinese Academy of Sciences, \\ 20A Datun Rd., Chaoyang District, 100012, Beijing, China \\ ${ }^{3}$ Astronomisches Rechen-Institut, Zentrum für Astronomie, University of Heidelberg, \\ Mönchhofstrasse 12-14, 69120, Heidelberg, Germany
}

\begin{abstract}
We compare the performance of the very popular Tree-GPU code BONSAI with the older Particle-(Multi)Mesh code SUPERBOX. Both code we run on a same hardware using the GPU acceleration for the force calculation. SUPERBOX is a particle-mesh code with high resolution sub-grid and a higher order NGP (nearest grid point) force-calculation scheme. In our research, we are aiming to demonstrate that the new parallel version of SUPERBOX is capable to do the high resolution simulations of the interaction of the system of disc-bulge-halo composed galaxy. We describe the improvement of performance and scalability of SUPERBOX particularly for the Kepler cluster (NVIDIA K20 GPU). A comparison was made with the very popular and publicly available Tree-GPU code BONSAI $\dagger$.
\end{abstract}

Keywords. $N$-body galaxy simulation, parallel computing, GPGPU, supercomputer

\section{Overview}

- Direct gravitational $N$-body simulations: computational complexity $\sim N^{2}$

* $\varphi$ GRAPE \& $\varphi$ GPU codes (Harfst et al. 2007; Berczik et al. 2011, 2013)

- Tree methods: computational complexity $\sim N \log (N)$

* bonsai GPU tree code (Bédorf et al. 2012)

- Particle mesh method: computational complexity $a N_{\text {grid }}^{3} \log \left(N_{\text {grid }}\right)+b N_{\text {part }}$

* SUPERBOX code (Fellhauer et al. 2000)

\section{Superbox code}

The time-step cycle of SUPERBOX is divided into two main routines ( $98 \%$ of the total CPU time) (Fellhauer 2006):

1 - the FFT routine computes the potential on the grids (MPI or OpenMP parallel part) 2 - the PUSHER routine contains the force calculation, the position and velocity updating and collection of the output data (serial part) .

\section{The SUPERBOX advantages}

- The method is fast. The method is self-consistent.

- Due to the large number of particles statistical noise is extremely low.

- Effects of two body relaxation are negligible

$\dagger$ http://castle.strw.leidenuniv.nl/software/bonsai-gpu-tree-code.html 

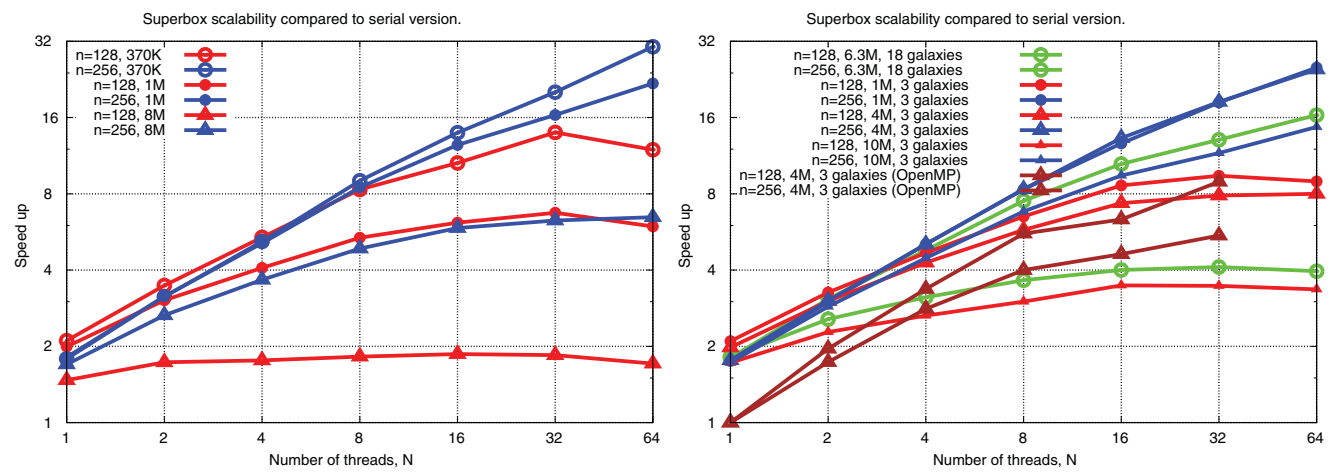

Figure 1. Scalability of parallel SUPERBOX version on Kepler cluster for single and multi galaxy models.

After optimization on Kepler cluster the parallel version of SUPERBOX show the increase of performance and good MPI scalability up to $64 \mathrm{CPU}$, for grid size $=256$ and up to $20 \mathrm{M}$ particles.

- OpenMP version have good scalability and need less memory, but is limited by maximum CPU cores

- The particle positions update module have bad speedup with OpenMP

\section{SUPERBOX vs BONSAI performance}

Calculation time for one integration step show comparable performance (Bien et al. 2008):

- SUPERBOX (8 MPI CPU, 4M particles, grid-size=128) 3.4s

- SUPERBOX (16-core OpenMP CPU, 4M particles, grid-size=128) 5.6s

- BONSAI2 (2x GTX 570 GPU, 4M particles, $\theta=0.5$ ) $4 \mathrm{~s}$

- BONSAI2 (Tesla 20X GPU, 10M particles, $\theta=0.75$ ) 1s

- SUPERBOX (32 MPI CPU, 10.2M particles, grid-size=128) 8.3s

\section{Acknowledgements}

OV \& PB thank the International Astronomical Union for the grant support which gave the opportunity for him to participate on the IAU Symposium No. 312 in Beijing2014 .

\section{References}

Berczik, P., Nitadori, K., Zhong, S., et al. 2011, in International conference on High Performance Computing, Kyiv, Ukraine, October 8-10, 2011., p. 8-18, 8-18

Berczik, P., Spurzem, R., Wang, L., Zhong, S., \& Huang, S. 2013, in Third International Conference "High Performance Computing", HPC-UA 2013, p. 52-59, 52-59

J. Bédorf, E. Gaburov, \& S. Portegies Zwart. Bonsai: A GPU Tree-Code. In R. CapuzzoDolcetta, M. Limongi, and A. Tornambè, editors, Advances in Computational Astrophysics: Methods, Tools, and Outcome, volume 453 of Astronomical Society of the Pacific Conference Series, page 325, July 2012.

R. Bien, A. Just, P. Berczik, \& I. Berentzen. High resolution in z-direction: The simulation of disc-bulge-halo galaxies using the particle-mesh code SUPERBOX. Astronomische Nachrichten, 329:1029, December 2008. 
M. Fellhauer, P. Kroupa, H. Baumgardt, R. Bien, C. M. Boily, R. Spurzem, \& N. Wassmer. SUPERBOX - an efficient code for collisionless galactic dynamics. New A, 5:305-326, September 2000 .

Fellhauer M., 2006, Superbox manual, madf@ast.cam.ac.uk

Harfst, S., Gualandris, A., Merritt, D., et al. 2007, New. Astr., 12, 357 